and clinical practice, we suggest a framework for the investigation of suspected recurrence cases (Fig. 1). However, the challenge of this detailed investigation lies in the high resource load required to allow truly robust conclusions, especially considering complicating factors in a postvaccination setting.

Nonetheless, we propose that studies that employ such techniques must be undertaken to appropriately answer the multitude of pressing questions that pertain to COVID-19 recurrence. These investigations are particularly significant considering recent reports of reinfection by novel SARS-CoV-2 variants, which may lead to a more severe second episode of disease. ${ }^{8}$ Thus, only when we comprehend the complex interplay between COVID-19 recurrence and the other components of the pandemic will we be able to quantify and react to its impact on both the patient and population levels.

Supplementary material. To view supplementary material for this article, please visit https://doi.org/10.1017/ice.2021.226

Acknowledgments. We thank Santiago Rene Uricochea Camacho, Marianna Cavina de Figueiredo, Gabriel Schier de Fraga, Roberta Garcia, Gustavo Pessatto Krause, André Luiz Parmegiani, Ana Júlia Pereira de Paula, Marcos Roberto Curcio Pereira, Gabriele Castro Schleuner, Fábio Augusto da Rocha Specian, Rebecca Benicio Stocco and Kimberlly Assumpção Vaz for the valuable discussions in our COVID-19 research group.

Financial support. Dr Viviane Maria de Carvalho Hessel Dias recieved financial support from the CAPES Foundation. The publication was funded by Pontíficia Universidade Católica do Paraná.

Conflicts of interest. All authors report no conflicts of interest relevant to this article.

\section{References}

1. Chen J, $\mathrm{Xu} \mathrm{X}, \mathrm{Hu} \mathrm{J}$, et al. Clinical course and risk factors for recurrence of positive SARS-CoV-2 RNA: a retrospective cohort study from Wuhan, China. Aging 2020;12:16675-16689.

2. Huang J, Zheng L, Li Z, et al. Kinetics of SARS-CoV-2 positivity of infected and recovered patients from a single center. Sci Rep 2020;10:18629.

3. Zheng J, Zhou R, Chen F, et al. Incidence, clinical course and risk factor for recurrent PCR positivity in discharged COVID-19 patients in Guangzhou, China: a prospective cohort study. PLOS Neglected Trop Dis 2020;14(8): e0008648.

4. Gousseff M, Penot P, Gallay L, et al. Clinical recurrences of COVID-19 symptoms after recovery: viral relapse, reinfection or inflammatory rebound? J Infect 2020;81:816-846.

5. To KK-W, Hung IF-N, Ip JD, et al. Coronavirus disease 2019 (COVID-19) reinfection by a phylogenetically distinct severe acute respiratory syndrome coronavirus 2 strain confirmed by whole genome sequencing. Clin Infect Dis 2020 Aug 25; ciaal275.

6. Morin L, Savale L, Pham T, et al for the COMEBAC Study Group. Four-month clinical status of a cohort of patients after hospitalization for COVID-19. JAMA 2021;325:1525-1534.

7. Jefferson T, Spencer EA, Brassey J, Heneghan C. Viral cultures for COVID-19 infectious potential assessment-a systematic review. Clin Infect Dis 2020. doi: 10.1093/cid/ciaal764.

8. Zucman N, Uhel F, Descamps D, Roux D, Ricard J-D. Severe reinfection with South African SARS-CoV-2 variant 501Y.V2: a case report. Clin Infect Dis 2021. doi: 10.1093/cid/ciab129.

9. Benner SE, Patel EU, Laeyendecker O, et al. SARS-CoV-2 antibody avidity responses in COVID-19 patients and convalescent plasma donors. J Infect Dis 2020;222:1974-1984.

10. Lu J, Peng J, Xiong Q, et al. Clinical, immunological and virological characterization of COVID-19 patients that test re-positive for SARSCoV-2 by RT-PCR. EBioMedicine 2020. doi: 10.1016/j.ebiom.2020. 102960 .

\title{
Coronavirus disease 2019 (COVID-19) outbreak on an inpatient psychiatry unit: Mitigation and prevention
}

\author{
Jamie M. McGloin BS ${ }^{1, a}$ (1), Nithila Asokaraj BSN, RN, ClC 2 ,a , Baevin Feeser MPH, CIC ${ }^{1}$, Barbara Carney MSN, $\mathrm{RN}^{3}$, \\ Kari Phillips MSN, RN³ , Liliana Smurawska $\mathrm{MD}^{3}$, Rohn S. Friedman $\mathrm{MD}^{3}$, Sharon B. Wright MD, $\mathrm{MPH}^{1,4}$ and \\ Dana E. Pepe MD, MPH ${ }^{1,4}$ \\ ${ }^{1}$ Division of Infection Control/Hospital Epidemiology, Silverman Institute for Health Care Quality and Safety, Beth Israel Deaconess Medical Center, Boston, \\ Massachusetts, ${ }^{2}$ Department of Hospital Epidemiology and Infection Prevention, University of California San Francisco, San Francisco, California, ${ }^{3}$ Department of \\ Psychiatry, Beth Israel Deaconess Medical Center, Boston, Massachusetts and ${ }^{4}$ Division of Infectious Diseases, Beth Israel Deaconess Medical Center, Boston, \\ Massachusetts
}

To the Editor-Severe acute respiratory coronavirus virus 2 (SARS-CoV-2) is primarily spread through respiratory droplets with increased risk of transmission in households and congregate settings. ${ }^{1-3}$ Asymptomatic and presymptomatic transmission of SARS-CoV-2 have also made containment difficult. ${ }^{1,4}$ Inpatient psychiatry units present unique challenges in controlling infectious disease outbreaks. ${ }^{5,6}$ Here, we describe the management of a

Author for correspondence: Jamie M. McGloin, E-mail: jmcgloin@bidmc.harvard.edu authors of equal contribution.

Cite this article: McGloin JM, et al. (2022). Coronavirus disease 2019 (COVID-19) outbreak on an inpatient psychiatry unit: Mitigation and prevention. Infection Control \& Hospital Epidemiology, 43: 1290-1291, https://doi.org/10.1017/ice.2021.233 coronavirus disease 2019 (COVID-19) outbreak on an inpatient psychiatry unit, highlighting unique considerations for this patient population.

Beth Israel Deaconess Medical Center (BIDMC) is a 655-bed, academic, tertiary-care center in Boston, Massachusetts, with a 25-bed inpatient psychiatry unit including multiple 2 - and 4-bed patient rooms and communal living spaces. In March 2020, Infection Control/Hospital Epidemiology (IC/HE) was notified of an asymptomatic inpatient with concern for COVID-19 due to a community exposure prior to admission. The index patient, who had been admitted to a double room the day prior (hospital day 0 ), was placed on precautions and 
Table 1. Inpatient Psychiatric Control Interventions Implemented during Outbreak

\begin{tabular}{|c|c|c|}
\hline General Patient Level & Environmental Level & Specific to the Psychiatric Population \\
\hline $\begin{array}{l}\text { - Surgical masks for all patients } \\
\text { - Surgical masks and eye protection for all } \\
\text { employees } \\
\text { - Symptom screening patient and staff } \\
\text { daily } \\
\text { - Increase patient compliance to hand } \\
\text { hygiene } \\
\text { - Restrict all visitors }\end{array}$ & $\begin{array}{l}\text { - Increase frequency of cleaning in } \\
\text { shared spaces } \\
\text { - Bleach cleaning daily } \\
\text { - Enhanced terminal cleaning: } \\
\text { O Change curtains } \\
\text { O Deep cleaning with bleach } \\
\text { O Ultraviolet (UV) light disinfection }\end{array}$ & $\begin{array}{l}\text { - Adhere to safety measures for patient masking (ie, no metal nose } \\
\text { clips or ties) } \\
\text { - Limit number of patients per group therapy session to five and } \\
\text { physically distance } \\
\text { - Stagger patient meal times } \\
\text { - Reduce shared patient supplies }\end{array}$ \\
\hline
\end{tabular}

was transferred to a medical floor for evaluation. Upon transfer, the patient further disclosed 14 days of dry cough and subsequently had 2 SARS-CoV-2 polymerase chain reaction (PCR) tests $\geq 12$ hours apart, per hospital protocol. The first test was negative and the second was positive on hospital day 3 . The next day, the index patient's former roommate reported headache and congestion. The roommate was placed on precautions, transferred, and tested negative twice. Two employees also reported symptoms that day but subsequently tested negative for SARS-CoV-2.

The remaining patients on the unit were screened each shift for symptoms including fever $\left(\mathrm{T}>38^{\circ} \mathrm{C}\right)$ or feverishness, new or worsening cough, sore throat, shortness of breath, diarrhea, vomiting, or severe fatigue. Employees were screened once daily. Nasopharyngeal swabs were obtained for SARS-CoV-2 PCR using the Abbott RealTime SARS-CoV-2 assay (Abbott Diagnostics, Abbot Park, IL) for all symptomatic patients and staff.

A multidisciplinary team consisting of medicine, psychiatry, and IC/HE leadership met daily to plan and implement additional control measures. These measures included closure to new admissions on hospital day 4, implementation of personal protective equipment (PPE), observed hand hygiene before meals and group therapy sessions, visitor restriction, and continued patient and staff symptom screening. PPE consisted of surgical masks and eye protection for staff and surgical masks for patients who were able to adhere. Psychiatry unit-specific measures to promote physical distancing involved limiting the number of patients in shared spaces and staggering group mealtimes. Environmental-focused interventions involved increased cleaning frequency and bleaching of communal spaces and shared equipment (Table 1).

The outbreak lasted for a total of 27 days, with the last cases confirmed on day 20. A median of 11 days was observed between date of exposure to index patient and positive test. Between days 5 and 20, 6 additional patients became symptomatic, and 4 tested positive. Also, 10 additional employees reported symptoms, and 5 of these tested positive. Notably, no patients or employees were retested after initial testing for symptom evaluation. Environmental services completed enhanced terminal cleaning of the unit (Table 1), 2 remaining exposed patients were transferred to the medicine service, and the unit was reopened to admissions on day 27. In total, 20 individuals reported COVID-19 symptoms; 5 (63\%) of 8 symptomatic patients and $5(42 \%)$ of 12 of symptomatic employees tested positive.

There were several limitations to implementing interventions. First, staff uptake of recommended PPE was slow initially, but it increased with further encouragement and observations from unit leadership and IC/HE. Additionally, complex patient behaviors affected proper PPE use, physical distancing adherence, and consistent symptom reporting. Physical distancing was also difficult given the fundamental practice of group sessions in patient treatment and the communal nature of the unit. Lastly, asymptomatic patients and staff were not tested at the time of this outbreak due to limited global testing capacity early in the pandemic. A more inclusive testing strategy may also be applied as testing capacity allows.

Acknowledgments.

Financial support. No financial support was provided relevant to this article.

Conflicts of interest. All authors report no conflicts of interest relevant to this article.

\section{References}

1. Transmission of SARS-CoV-2: implications for infection prevention practices. World Health Organization website. https://www.who.int/news-room/ commentaries/detail/transmission-of-sars-cov-2-implications-for-infectionprevention-precautions. Accessed April 28, 2021.

2. Zhang J, Tian S, Lou J, Chen Y. Familial cluster of COVID-19 infection from an asymptomatic. Crit Care 2020;24:119.

3. McMichael TM, Currie DW, Clark S, et al. Epidemiology of COVID-19 in a long-term-care facility in King County, Washington. N Engl J Med. 2020; 382:2005-2011.

4. Gandhi M, Yokoe DS, Havlir DV. Asymptomatic transmission, the Achilles' heel of current strategies to control COVID-19. N Engl J Med 2020;382: 2158-2160.

5. Weber DJ, Sickbert-Bennett EE, Vinje J, et al. Lessons learned from a norovirus outbreak in a locked pediatric inpatient psychiatric unit. Infect Control Hosp Epidemiol 2005;26:841-843.

6. Johnston CP, Qiu H, Ticehurst JR, et al. Outbreak management and implications of a nosocomial norovirus outbreak. Clin Infect Dis 2007;45:534-540. 